\title{
Cost-effectiveness of immediate versus delayed sequential bilateral cataract surgery in the Netherlands (the BICAT-NL study): study design of a prospective multicenter randomised controlled trial
}

\author{
L. S. Spekreijse ${ }^{1,2^{*}}$ (D) R. W. P. Simons ${ }^{1}$, B. Winkens ${ }^{3}$, F. J. H. M. van den Biggelaar ${ }^{1}$, C. D. Dirksen ${ }^{4}$ and \\ R. M. M. A. Nuijts ${ }^{1,5}$
}

\begin{abstract}
Background: Cataract surgery is one of the most frequently performed types of surgery. Most patients suffer from bilateral cataract and while cataract surgery of only one eye is effective in restoring functional vision, second-eye surgery leads to further improvements in health-related quality of life, and is cost-effective. At present, most patients undergo cataract surgery in both eyes on separate days as recommended in national guidelines, referred to as delayed sequential bilateral cataract surgery (DSBCS). An alternative procedure involves operating both eyes on the same day, but as separate procedures, known as immediately sequential bilateral cataract surgery (ISBCS). The aim of this study is to evaluate the effectiveness and costs of ISBCS compared to DSBCS, in order to test the hypothesis that ISBCS is non-inferior to DSBCS in terms of effectiveness and superior to ISBCS in terms of costeffectiveness.
\end{abstract}

Methods/design: Multicenter non-inferiority randomised controlled clinical trial. Patients (18 years or older) with bilateral cataract and an indication for bilateral cataract surgery with an expected uncomplicated intraoperative and postoperative course are included in the study. Patients are randomly assigned to either ISBCS or DSBCS. The primary endpoint is the proportion of patients with a refractive outcome in the second eye within 1.0 dioptre from the target refraction, at 4 weeks after surgery. Secondary outcomes include corrected and uncorrected distance visual acuity, complications, patient reported outcomes (PROMs), cost-effectiveness, and budget impact. Follow-up visits are planned at 1 week after first-eye surgery and 4 weeks after second-eye surgery. At 3 months after first-eye surgery, the occurrence of complications is checked and patients fill in a final questionnaire.

(Continued on next page)

\footnotetext{
* Correspondence: lindsay.spekreijse@mumc.nl

${ }^{1}$ Maastricht University Medical Center+, University Eye Clinic Maastricht, P. Debyelaan 25, 6229 HX Maastricht, the Netherlands

${ }^{2}$ School for Mental Health and Neuroscience (MHeNs), Maastricht University, Maastricht, the Netherlands

Full list of author information is available at the end of the article
}

(c) The Author(s). 2020 Open Access This article is licensed under a Creative Commons Attribution 4.0 International License, which permits use, sharing, adaptation, distribution and reproduction in any medium or format, as long as you give appropriate credit to the original author(s) and the source, provide a link to the Creative Commons licence, and indicate if changes were made. The images or other third party material in this article are included in the article's Creative Commons licence, unless indicated otherwise in a credit line to the material. If material is not included in the article's Creative Commons licence and your intended use is not permitted by statutory regulation or exceeds the permitted use, you will need to obtain permission directly from the copyright holder. To view a copy of this licence, visit http://creativecommons.org/licenses/by/4.0/ The Creative Commons Public Domain Dedication waiver (http://creativecommons.org/publicdomain/zero/1.0/) applies to the data made available in this article, unless otherwise stated in a credit line to the data. 
(Continued from previous page)

Discussion: This study protocol describes the design of a multicenter non-inferiority randomised controlled trial. Current studies on ISBCS often lack information on safety regarding refractive outcomes. In addition, there is a lack of well-designed cost-effectiveness studies using established methods. The BICAT-NL study will provide more insight in refractive and cost-effectiveness outcomes for ISBCS compared to DSBCS.

Trial registration: This study was prospectively registered at Clinicaltrials.gov on January 17th 2018. (Identifier: NCT03400124.

Keywords: Immediately sequential bilateral cataract surgery (ISBCS), Delayed sequential bilateral cataract surgery (DSBCS), Randomized controlled trial, Cost-effectiveness, Refraction, Complications, Visual acuity, Patient reported outcome measures (PROMs)

\section{Background}

Cataract surgery is one of the most frequently performed types of surgery in the world. In the Netherlands, the current estimated number of cataract extractions is over 180,000 per year $[1,2]$. This number has increased rapidly over the years. As a result, national healthcare expenditures on cataract have increased as well. Due to the vast number of cataract patients, small efficiency gains in cataract care delivery may lead to substantial cost savings on a macro level.

The majority of patients, mostly elderly, suffer from bilateral cataract. A previous study showed that $71 \%$ of patients with cataract had an indication for bilateral surgery [3]. While cataract surgery of only one eye is effective in restoring functional vision, studies have shown that cataract surgery of the second eye leads to further improvements in health-related quality of life, and is cost-effective [4-6].

At present, most patients undergo cataract surgery in both eyes on separate days, as advised in the Cataract guidelines of the Dutch Ophthalmology Society, with a delay of at least 2 weeks (delayed sequential bilateral cataract surgery, DSBCS) [7]. However, over the past years some have argued that the procedure is now safe enough to perform in both eyes on the same day during a single operating session (immediately sequential bilateral cataract surgery, ISBCS) [8]. These advocates substantiate their attitudes to ISBCS through the many advances in the field of cataract surgery and consider ISBCS to be safe when risks of complications related to surgical procedures are minimized [9].

The two primary reasons for delaying second-eye surgery are the risk of bilateral endophthalmitis and the risk of refractive surprise. Endophthalmitis is most likely to occur within the first 2 weeks after surgery. When this happens, cataract surgeons may decide to abstain from second-eye surgery. However, unilateral endophthalmitis is a rare complication, especially since the introduction of intracameral antibiotic prophylaxis. Several retrospective and epidemiologic studies have reported that the administration of intracameral antibiotics significantly reduces the risk for developing endophthalmitis compared to other prophylactic measures, such as topical antibiotics $[10,11]$. More recent studies on incidences of endophthalmitis after administration of intracameral antibiotics, show endophthalmitis rates of $0.039 \%$ (Spain) [12] and 0.029\% (Sweden) [13]. In addition, the study on Swedish national data reports a significant decrease in endophthalmitis rates compared to previous years [13]. The calculated probability of endophthalmitis occurring bilaterally is extremely low (1: 70 million cases) [8]. So far, the few cases of bilateral endophthalmitis after ISBCS that have been described could be attributed to faults in aseptic procedures [14]. Three randomised controlled trials (RCTs) [15-17] and several non-comparative studies [18-26] found no significant differences in (severe) complication rates. With regard to refractive outcomes, delaying second-eye surgery enables cataract surgeons to evaluate the outcomes of the first eye and, if necessary, adjust their plans for second-eye surgery [27]. In the Netherlands, the standard for success that is used in cataract surgery is a postoperative refraction within 1.0 diopter of the target refraction [28]. Only two previous studies, one randomised study and one nonrandomised comparative study, [15, 29] report data on refractive outcomes for ISBCS compared to DSBCS. These studies showed similar refractive outcomes for ISBCS compared to DSBCS, indicating that refractive surprises may be prevented with careful patient selection $[15,25,29,30]$. However, the number of patients in these two studies was limited and the overall quality of the evidence of the RCT was graded low to moderate [31]. Therefore, concerns remain with regard to refractive outcomes.

Furthermore, available studies on cost analyses showed that ISBCS resulted in fewer costs and important cost savings to third-party payers, patients, and society compared to DSBCS [3, 32-36]. However, to date, only one (modelbased) cost-utility analysis has been performed [36].

Therefore, the aim of the BICAT-NL (Bilateral Cataract surgery in the Netherlands) study is to evaluate the effectiveness and costs of ISBCS compared to DSBCS in the Netherlands in order to test the hypotheses that 
ISBCS is non-inferior to DSBCS in terms of effectiveness and superior to ISBCS in terms of cost-effectiveness.

\section{Methods/design \\ Objectives}

The primary objective of this study is to evaluate whether ISBCS is non-inferior to DSBCS regarding effectiveness, where effectiveness is defined as the proportion of patients with a postoperative refraction that deviates $\leq 1 \mathrm{D}$ from target refraction. Secondary objectives of this study are to evaluate ISBCS versus DSBCS regarding non-inferiority of (1) the proportion of patients with a postoperative refraction within $0.5 \mathrm{D}$ of target refraction, and (2) postoperative visual acuity, and superiority of (3) patient satisfaction (using patient reported outcome measures (PROMs)), (4) the incidence of complications, and (5) cost-effectiveness.

\section{Study design and setting}

A multicenter non-inferiority randomized controlled clinical trial will be performed at outpatient ophthalmology clinics in one academic center (Maastricht University Medical Center (MUMC+)) and nine non-academic centers in the Netherlands (Zuyderland Medical Center, Heerlen; Canisius Wilhelmina Hospital, Nijmegen; Gelre Hospital, Zutphen; Deventer Hospital, Deventer; Elisabeth TweeSteden Hospital, Tilburg; Amphia Hospital, Breda; Medical Center Haaglanden, Den Haag; Medical Spectrum Twente, Enschede; Isala Clinic, Zwolle).

\section{Study population}

All patients with bilateral cataracts undergoing expected uncomplicated bilateral cataract surgery using a standard phacoemulsification technique will qualify for inclusion in this study. The exclusion criteria are listed in Fig. 1.

\section{Study procedures}

Visits are planned at baseline (preoperatively), and 1 week after first-eye surgery and 4 weeks after second-eye surgery. Patients will undergo study procedures as shown in Table 1. At 3 months after surgery, the occurrence of complications is derived from the patient record

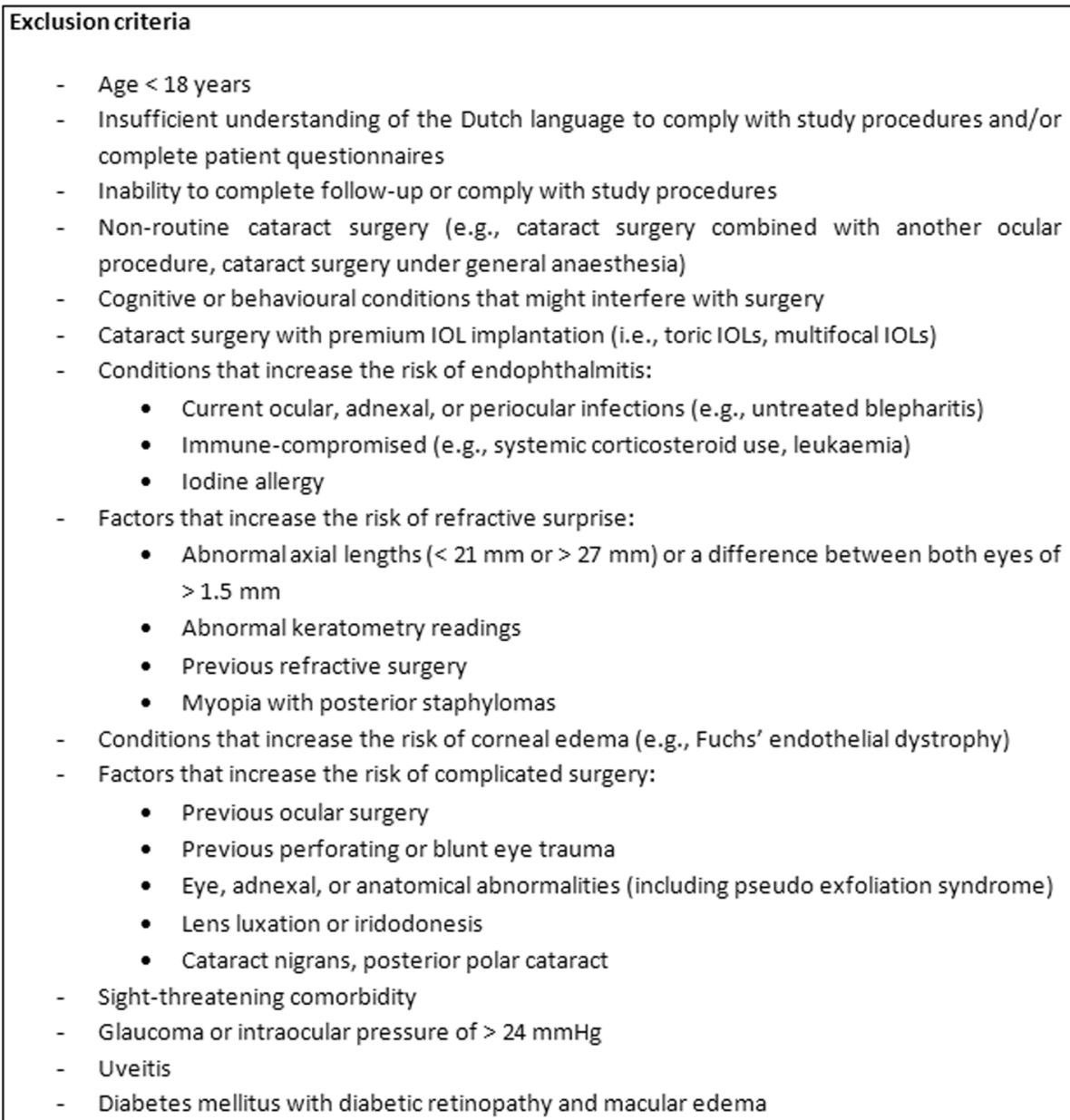

Fig. 1 Exclusion criteria 
Table 1 Study procedures per follow-up time point

\begin{tabular}{|c|c|c|c|c|c|c|}
\hline \multirow[t]{2}{*}{ Assessment/ procedure } & \multicolumn{6}{|l|}{ Visit } \\
\hline & Preoperative & Surgery 1 & 1 week after surgery 1 & Surgery 2 (if applicable) & $\begin{array}{l}4 \text { weeks after } \\
\text { surgery } 2\end{array}$ & $\begin{array}{l}3 \text { months after surgery } 1 \\
\text { (post/email) }\end{array}$ \\
\hline Check for in-/exclusion criteria & $x$ & & & & & \\
\hline Informed Consent & $x$ & & & & & \\
\hline Medical history & $x$ & & & & & \\
\hline Check medication & $x$ & $x$ & $x$ & $x$ & $x$ & $x^{a}$ \\
\hline Ophthalmic examination & $x$ & & $x$ & & $x$ & \\
\hline Check adverse events & & $x$ & $x$ & $x$ & $x$ & $x^{a}$ \\
\hline Refraction & $x$ & & $x$ & & $x$ & \\
\hline CDVA & $x$ & & $x$ & & $x$ & \\
\hline NEI VFQ-25 questionnaire & $x$ & & & & & $x$ \\
\hline Catquest-9SF questionnaire & $x$ & & & & & $x$ \\
\hline EQ-5D-5L questionnaire & $x$ & & $x$ & & $x$ & $x$ \\
\hline HUI-3 questionnaire & $x$ & & $x$ & & $x$ & $x$ \\
\hline Cost questionnaire & $x$ & & & & & $x$ \\
\hline
\end{tabular}

${ }^{a}$ derived from the patient record and questionnaires

and patients will fill in the final questionnaires. Visual acuity will be measured by using the Early Treatment Diabetic Retinopathy Study (ETDRS) chart and measured refraction will be subjective (not automated).

The intervention consists of bilateral cataract surgery by an experienced cataract surgeon. A standard phacoemulsification technique with intraocular lens implantation will be used. Surgery will be performed either during a single operating session (ISBCS) or during separate operating sessions with a minimum of 2 weeks apart (DSBCS), depending on randomisation. ISBCS will be performed in accordance with the "iSBCS General Principles for Excellence in ISBCS 2009", treating both eyes as completely separate aseptic procedures in order to minimize the risk of bilateral endophthalmitis. For both ISBCS and DSBCS, intracameral antibiotics will be administered at the end of each operation. Topical antibiotics and anti-inflammatory drugs will be commenced postoperatively. After surgery, DSBCS patients will receive an eye patch and ISBCS patients will receive a transparent eye shield.

Patients can withdraw from the study at any time for any reason without any consequences. The investigator can decide to withdraw a patient from the study for urgent medical reasons. In case a patient withdraws from the study after randomization, the case is considered lost to follow-up, but will be included in the intention to treat analysis.

\section{Outcome measures}

The primary outcome is the proportion of patients in both treatment groups with a postoperative refraction in the second eye that deviates $\leq 1.0 \mathrm{D}$ from target refraction 4 weeks after cataract surgery of the second eye. This is an accepted norm to define success in terms of refractive outcomes after cataract surgery [7, 28].

Secondary outcomes will include:

1. Refraction: the proportion of patients in both treatment groups with a postoperative refraction in the second eye that deviates $\leq 0.5 \mathrm{D}$ from target refraction 4 weeks after cataract surgery of the second eye.

2. Visual acuity: ETDRS, uncorrected and corrected distance visual acuity in LogMAR (the Logarithm of the Minimum Angle of Resolution), 1 week after first-eye surgery and 4 weeks after second-eye surgery, corrected for baseline visual acuity. In addition, the proportion of patients with a final corrected distance visual acuity (CDVA) of $\leq 0.1$ LogMAR (measured at 4 weeks after second-eye surgery) will be compared for ISBCS and DSBCS.

3. Complications: the number of intraoperative and postoperative complications within 3 months after first-eye surgery.

4. Patient-reported outcome measures (PROMs), including patient satisfaction, vision-specific quality of life, and health-related quality of life:

a. Vision-specific quality of life and patient satisfaction will be measured using the National Eye Institute Visual Function Questionnaire-25 (NEI VFQ-25) [37] and the Catquest-9SF [38], measured at baseline and 3 months after firsteye surgery (Table 1 ).

b. Health-related quality of life (HRQL) will be measured using two questionnaires: EuroQol's 
EQ-5D-5L [39] and the Health Utilities Index Mark-3 (HUI-3) [40], measured at baseline, 1 week after first-eye surgery, 4 weeks after second-eye surgery and 3 months after first-eye surgery (Table 1).

5. Incremental cost-effectiveness ratios (ICERs): these will be expressed as 1) incremental societal costs per quality-adjusted life year (QALY) gained, 2) incremental healthcare costs per patient with postoperative refraction $\leq 1.0 \mathrm{D}$ from target refraction, 3) incremental healthcare costs per clinically improved patient on the NEI VFQ-25 questionnaire, 4) incremental healthcare costs per clinically improved patient on the Catquest-9SF questionnaire, and 5) incremental healthcare costs per patient with clinical improvement in (un)corrected distance visual acuity. QALYs will be calculated based on generic HRQL.

6. Budget impact will be reported as a difference in costs. Different scenarios will be compared to investigate the impact of various levels of implementation.

Adverse events are defined as any undesirable ophthalmic event occurring during the study, whether or not considered related to the trial procedure or the experimental intervention. All ophthalmic adverse events reported spontaneously by the patient or observed by the investigator or his staff will be recorded. Serious adverse events are defined as any untoward medical occurrence or effect that results in death, is life threatening (at the time of the event), requires hospitalisation or prolongation of existing inpatients' hospitalisation, results in persistent or significant disability or incapacity, is a congenital anomaly or birth defect, or any other important medical event that did not result (but could have resulted) in any of the outcomes listed above due to medical or surgical intervention.

\section{Randomisation and blinding}

Study participants will be randomized to either ISBCS (intervention) or DSBCS (control). Each patient will receive a randomization number from a computerized random number generator. In a certified electronic data capture tool called Castor EDC [41], the subject number will be allocated to ISBCS or DSBCS through block randomisation stratified for center and axial length. Random varying block sizes of 2 and 4 will be used. Blinding is not possible because of the nature of the intervention.

\section{Sample size calculation}

The sample size calculation is based on the proportion of patients with a postoperative refractive error in the second eye that deviates less than $1.0 \mathrm{D}$ from target refraction. The only RCT thus far that reported this outcome showed that 91.0 and $90.3 \%$ of ISBCS and DSBCS eyes, respectively, was within 1.0 D [15]. Another (nonrandomized) comparative study showed that 96.8 and $97.0 \%$ of ISBCS and DSBCS eyes, respectively, was within $1.0 \mathrm{D}$ [29]. Since no difference is expected with regard to this outcome, these percentages were averaged giving an expected proportion of $94 \%$ in both treatment groups. For the sample size calculation, it is assumed that the proportion in the ISBCS group is equal to or smaller than the proportion in the DSBCS group (i.e., non-inferiority). A non-inferiority margin of $5 \%$ is allowed (i.e., the proportion in ISBCS would need to be $>5 \%$ lower than the proportion in DSBCS to detect a statistically significant difference). The probability of a Type I error (alpha) is set at 0.05 , and the probability of a Type II error (beta) is set at 0.10 (i.e., power is $90 \%$ ). All sample sizes were calculated using an online 2sample non-inferiority or superiority calculator for the comparison of two proportions (www.powerandsamplesize.com). The required sample size is 386 patients per group or 772 patients in total. Factoring in a loss to follow-up of $10 \%$ gives a final sample size of 858 patients.

\section{Statistical analysis}

All data will be collected in a database (Castor) and will be exported to IBM SPSS Statistics (IBM, Armonk, NY, USA) for data analysis. The data analyses will be performed according to the intention to treat principle. However, considering that second-eye surgery can be delayed in ISBCS patients with complications during firsteye surgery, the analyses will be repeated based on a per protocol analysis. As recommended for non-inferiority trials in literature, both analyses will be performed and non-inferiority will be established if both analyses produce the same conclusion [42]. For all analyses, stratification variables (center and axial length) are accounted for by including them in the analysis model and significance levels will be set at 0.05 .

\section{Baseline characteristics}

Baseline characteristics will be presented either as means with standard deviation and $95 \%$ confidence intervals, as median with interquartile range, or as frequencies (with percentages), as appropriate.

\section{Primary outcome}

With regard to the primary endpoint of refractive outcome, the difference in the proportion of second eyes with a refractive outcome $\leq 1.0 \mathrm{D}$ of target refraction will be analysed using a logistic regression analysis with correction for stratification variables (center and axial length), where a logistic mixed model with center as 
random factor to account for the hierarchical nature of the data (patients are nested in centers) will be used as sensitivity analysis. To evaluate non-inferiority, a onesided test procedure will be used. Non-inferiority will be established at the $\alpha=0.05$ significance level if the lower limit of a $(1-2 \alpha) \times 100 \%$ confidence interval $(=90 \% \mathrm{CI})$ for the difference (ISBCS - DSBCS) is above the noninferiority margin $(\delta)$ of $-5 \%$ [42]. Possible scenarios of observed treatment differences between ISBCS and DSBCS will be evaluated as reported by the CONSORT Statement 2010 [43].

\section{Secondary outcomes}

For secondary outcomes, data analyses will be performed as follows: presence of non-inferiority of ISBCS compared to DSBCS regarding the difference in the proportion of second eyes with a refractive outcome within \pm $0.5 \mathrm{D}$ of target refraction will be evaluated similar to the method described under primary outcome. For visual acuity, presence of non-inferiority of ISBCS will be evaluated by comparing the proportion of patients with a corrected distance visual acuity (CDVA) 4 weeks after surgery of the second eye of $\leq 0.10$ LogMAR between groups. The computed sample size of 858 patients for the primary outcome translates to an allowed noninferiority margin of $10 \%$ to establish non-inferiority at the 0.05 alpha level regarding this secondary outcome measure. Corrected and uncorrected distance visual acuity in second eyes, patient-reported outcomes, and utilities will be analysed using a linear mixed model to test for overall differences between groups over time. The difference in the incidence of intraoperative and postoperative complications will be analysed using mixedeffects logistic regression to account for the fact that both eyes of each patient are included in the analysis and nesting of patients within centers.

\section{Cost-effectiveness analysis}

A trial-based economic evaluation will be performed over a three-month time horizon (first-eye surgery until the final questionnaires at 3 months after first-eye surgery). A societal and healthcare perspective will be used and ICERs will be calculated for the outcome measures described earlier in this protocol. Uncertainty in the point estimates of the ICERs will be assessed using bootstrap analyses with 1000 replications. Based on these bootstrap analyses, cost-effectiveness acceptability curves will be constructed, to show the probability that the intervention is cost-effective for a range of threshold values of the ICER. Additional sensitivity analyses will be performed to investigate the impact of varying input parameters used in the cost-effectiveness analysis.

In addition to the trial-based economic evaluation, the lifetime economic impact of ISBCS compared to DSBCS will be investigated by means of a decision analytical model, in order to take rare events (e.g. bilateral complications) into account. The model-based economic evaluation will address the cost per QALY (societal perspective) and cost per patient with postoperative refraction within 1.0 D from target refraction (health care perspective). Input data for the model will be based on the data from the current study and from a literature review. We will express the model's robustness and uncertainty by means of sensitivity analyses, confidence intervals, and by creating cost-effectiveness acceptability curves. Annual discount rates of 4 and $1.5 \%$ for costs and QALYs, respectively, will be used to determine the present value of costs and QALYs accrued over a lifetime.

In accordance with the Dutch guidelines for economic evaluations, the EQ-5D-5L will be used to determine QALYs using published Dutch tariffs [39]. In addition, the HUI-3 will be used because it is the only generic preference-based HRQL questionnaire that includes questions about vision [40].

For the cost analysis, all relevant costs incurred during the follow-up period from a healthcare and societal perspective will be included. Hospital-based resource use will be identified through patients records. Other resource use (e.g., use of homecare, costs of spectacles, productivity losses) will be measured through the standardized cost questionnaire at baseline and at 3 months after first-eye surgery. Resource use will be valued in accordance with the Dutch guidelines for costs analyses [44]. All costs will be adjusted for inflation to a common price level using the Consumer Price Index.

\section{Budget impact analysis}

A budget impact analysis (BIA) will be performed to evaluate the impact of implementation of ISBCS on the Dutch healthcare budget. The BIA will be performed in accordance with the Dutch guidelines for economic evaluations and the ISPOR guidelines $[45,46]$. The analysis will make use of the budget holder perspective. Additional perspectives include healthcare providers and insurers. A time horizon of 3 years will be used to account for gradual implementation of ISBCS. The BIA will be performed using a simple Microsoft Excel decision analytic model. It will be assumed that the difference in costs will be related completely to substitution of DSBCS by ISBCS. Different scenarios will be compared to investigate various levels of implementation (i.e., 25, 50, 75, and $100 \%$ of eligible patients). Sensitivity analyses will be performed to test the robustness of the analysis.

\section{Data management and monitoring}

Personal data will be handled confidentially, according to Good Clinical Practice guidelines (GCP). Data will be 
collected in an online case report form (CRF), available in the certified electronic data capture tool 'Castor' by a member of the study team of each study center. All data will be stored and analysed using only anonymous randomization numbers. Personal data connecting a patient to the anonymous number will be archived by the principal investigator of each study center for a period of 15 years. This study will be monitored by the Clinical Trial Center Maastricht (CTCM) in order to protect patient rights and accuracy of reported trial data. The CTCM is an academic research organisation, familiar with monitoring procedures.

\section{Discussion}

Worldwide, an increasing number of cataract surgeries is performed over the years and healthcare expenditures are rising. Therefore, small efficiency gains in cataract care delivery, such as those potentially gained in ISBCS, may lead to substantial cost savings on a macro level. ISBCS is increasingly practiced on a routine basis in a number of countries around the world, including Finland, certain regions in Spain, and Canada [47]. Potential benefits of ISBCS include less time between surgeries, fewer hospital visits, a faster total recovery period due to simultaneous postoperative care (eye drops) in both eyes and less use of homecare. As a consequence of the recent COVID-19 crisis we now face a scenario where the number of hospital visits, if feasible, should be decreased to diminish the risk for contamination in the hospital. ISBCS could be an instrument to significantly decrease residence time of patients in the clinic for surgery as well as for postoperative controls. However, opponents of ISBCS argue that the potential risks of severe bilateral complications and unexpected refractive outcomes supersede any economic arguments [48]. The main concerns about ISBCS include potential risks of bilateral complications of cataract surgery, most importantly the very rare but severe risk of endophthalmitis and the risk of refractive surprise (a significant deviation from the predicted refraction).

Further investigation is required to evaluate how ISBCS compares to DSBCS with respect to cataract surgery outcomes, in particular with regard to refractive outcomes. In addition, there is a lack of welldesigned cost-effectiveness studies using standard methods. The BICAT-NL study will provide more insight in refractive and cost-effectiveness outcomes for ISBCS compared to DSBCS.

\section{Study status}

Currently, recruitment of patients is on-going. Recruitment has started on the 4th of September 2018 and is expected to be completed by August 2020. The latest version of the study protocol is version 7.0 (date: 02-08-2019).

\section{Abbreviations}

BIA: Budget impact analysis; CDVA: Corrected Distance Visual Acuity; CRF: Case Report Form; CTCM: Clinical Trial Center Maastricht; DSBCS : Delayed Sequential Bilateral Cataract Surgery; ETDRS: Early Treatment Diabetic Retinopathy Study; GCP: Good Clinical Practice; HRQL: Health-related Quality of Life; HUI-3: Health Utility Index Mark 3; ICER(s): Incremental costeffectiveness ratio(s); ISBCS: Immediately Sequential Bilateral Cataract Surgery; LogMAR: Logarithm of the Minimum Angle of Resolution; NEI VFQ-

25: National Eye Institute Visual Function Questionnaire-25; PROM(s): Patient Reported Outcome Measure(s); QALY(s): Quality Adjusted Life Year(s); RCT: Randomised Controlled Trial

\section{Acknowledgements \\ Not applicable.}

Authors' contributions

LS developed the protocol for the BICAT-NL study that received ethical approval and wrote the protocol manuscript. RS wrote the initial grant application in cooperation with RN, FB and CD. RS wrote the cost-effectiveness section of the protocol. BW critically revised and contributed to the statistical analysis section. All authors critically revised the manuscript and approved the final version.

\section{Funding}

This study was supported by a research grant from the ZonMw Doelmatigheidsonderzoek program (Zorgevaluatie 30 aandoeningen K\&D; Grant number: 843004111$)$. The study protocol was reviewed by the funding body before allocating the grant.

Availability of data and materials

This manuscript does not contain any data or results. Therefore, data sharing is not applicable.

\section{Ethics approval and consent to participate}

This study has been approved by the medical ethics committee Maastricht University Medical Center+ and Maastricht University (azM/UM) (identifier: 172048) and will be conducted according to the principles of the Declaration of Helsinki and according to GCP guidelines. Furthermore, the study will be conducted in accordance with Dutch law. Participation of each study site has been locally approved by local review boards. This study was prospectively registered at Clinicaltrials.gov on January 17th 2018. (Identifier: NCT03400124). All participating patients are requested to sign informed consent after the nature of the study and possible risks have been fully explained.

\section{Consent for publication}

Not applicable.

\section{Competing interests}

The authors declare that they have no competing interests.

\section{Author details}

${ }^{1}$ Maastricht University Medical Center+, University Eye Clinic Maastricht, P. Debyelaan 25, 6229 HX Maastricht, the Netherlands. ${ }^{2}$ School for Mental Health and Neuroscience (MHeNs), Maastricht University, Maastricht, the Netherlands. ${ }^{3}$ Department of Methodology and Statistics, Faculty of Health, Medicine and Life Sciences (FHML), Care and Public Health Research Institute (CAPHRI), Maastricht University, Maastricht, the Netherlands. ${ }^{4}$ Department of Clinical Epidemiology and Medical Technology Assessment, CAPHRI School for Public Health and Primary Care, Maastricht University Medical Center+, Maastricht, the Netherlands. ${ }^{5}$ Department of Ophthalmology, Zuyderland Medical Center, Heerlen, the Netherlands.

Received: 29 May 2020 Accepted: 17 June 2020

Published online: 29 June 2020

\section{References}

1. Lundstrom $M$, et al. The changing pattern of cataract surgery indications: a 5-year study of 2 cataract surgery databases. Ophthalmology. 2015;122(1): $31-8$. 
2. Henry Y. Practice styles and preferences of Dutch cataract and refractive surgeons, 2015 survey. Maastricht: The Netherlands Ophthalmological Society Annual Meeting; 2015.

3. Neel ST. A cost-minimization analysis comparing immediate sequentia cataract surgery and delayed sequential cataract surgery from the payer, patient, and societal perspectives in the United States. JAMA Ophthalmol. 2014;132(11):1282-8

4. Frampton $\mathrm{G}$, et al. The clinical effectiveness and cost-effectiveness of second-eye cataract surgery: a systematic review and economic evaluation. Health Technol Assess. 2014;18(68):1-205 v-vi.

5. Chew M, et al. The impact of cataract, cataract types, and cataract grades on vision-specific functioning using rasch analysis. Am J Ophthalmol. 2012; 154(1):29-38.

6. Shekhawat NS, et al. Impact of first eye versus second eye cataract surgery on visual function and quality of life. Ophthalmology. 2017;124(10):1496503.

7. Nederlands Oogheelkundig Gezelschap. Richtlijn cataract; 2013. [Cited 2017 2017/09/06]; Available from: https://www.oogheelkunde.org/richtlijnen-ebmen-overige.

8. Arshinoff SA. Same-day cataract surgery should be the standard of care for patients with bilateral visually significant cataract. Surv Ophthalmol. 2012; 57(6):574-9.

9. The International Society of Bilateral Cataract Surgeons. The iSBCS general principles for excellence in ISBCS 2019; 2009. Available from: https://isbcs. org/.

10. Barry P, et al. ESCRS study of prophylaxis of postoperative endophthalmitis after cataract surgery: preliminary report of principal results from a European multicenter study. J Cataract Refract Surg. 2006;32(3):407-10.

11. Grzybowski A, et al. Commonly used Intracameral antibiotics for Endophthalmitis prophylaxis: a literature review. Surv Ophthalmol. 2020; S0039-6257(20)30072-2.

12. Rodriguez-Caravaca $\mathrm{G}$, et al. Incidence of endophthalmitis and impact of prophylaxis with cefuroxime on cataract surgery. J Cataract Refract Surg. 2013;39(9):1399-403

13. Friling $E$, et al. Six-year incidence of endophthalmitis after cataract surgery: Swedish national study. J Cataract Refract Surg. 2013;39(1):15-21.

14. Arshinoff SA, Odorcic S. Same-day sequential cataract surgery. Curr Opin Ophthalmol. 2009;20(1):3-12.

15. Sarikkola $A U$, et al. Simultaneous bilateral versus sequential bilateral cataract surgery: Helsinki simultaneous bilateral cataract surgery study report 1. J Cataract Refract Surg. 2011;37(6):992-1002.

16. Serrano-Aguilar $P$, et al. Immediately sequential versus delayed sequential bilateral cataract surgery: safety and effectiveness. J Cataract Refract Surg. 2012;38(10):1734-42.

17. Lundstrom $M$, et al. Benefit to patients of bilateral same-day cataract extraction: randomized clinical study. J Cataract Refract Surg. 2006;32(5): 826-30

18. Sarikkola AU, et al. Simultaneous bilateral cataract surgery: a retrospective survey. J Cataract Refract Surg. 2004;30(6):1335-41.

19. Johansson B. Resulting refraction after same-day bilateral phacoemulsification. J Cataract Refract Surg. 2004;30(6):1326-34.

20. Johansson BA, Lundh BL. Bilateral same day phacoemulsification: 220 cases retrospectively reviewed. Br J Ophthalmol. 2003;87(3):285-90,

21. Arshinoff SA, Strube YN, Yagev R. Simultaneous bilateral cataract surgery. J Cataract Refract Surg. 2003;29(7):1281-91.

22. Wertheim M, Burton R. Immediately sequential phacoemulsification performed under topical anaesthesia as day case procedures. $\mathrm{Br} \mathrm{J}$ Ophthalmol. 2002;86(12):1356-8.

23. Akçay $L$, et al. Simultaneous bilateral phacoemulsification and intraocular lens implantation. TJO. 2013;43(3):149-55.

24. Ganesh S, Brar S, Sreenath R. Immediate sequential bilateral cataract surgery: a 5-year retrospective analysis of 2470 eyes from a tertiary care eye center in South India. Indian J Ophthalmol. 2017;65(5):358-64.

25. Herrinton $L$, et al. Immediate sequential vs. delayed sequential bilateral cataract surgery: retrospective comparison of postoperative visual outcomes. Ophthalmology. 2017;124(8):1126-35.

26. Arshinoff SA, Bastianelli PA. Incidence of postoperative endophthalmitis after immediate sequential bilateral cataract surgery. J Cataract Refract Surg. 2011;37(12):2105-14

27. Olsen T. Use of fellow eye data in the calculation of intraocular lens power for the second eye. Ophthalmology. 2011;118(9):1710-5.
28. Lundstrom $M$, et al. Risk factors for refractive error after cataract surgery: analysis of 282811 cataract extractions reported to the European registry of quality outcomes for cataract and refractive surgery. J Cataract Refract Surg. 2018;44(4):447-52

29. Chung JK, et al. Bilateral cataract surgery: a controlled clinical trial. Jpn J Ophthalmol. 2009:53(2):107-13.

30. Nassiri N, et al. Immediate vs delayed sequential cataract surgery: a comparative study. Eye (Lond). 2009;23(1):89-95.

31. Kessel L, et al. Immediate sequential bilateral cataract surgery: a systematic review and meta-analysis. J Ophthalmol. 2015;2015:912481.

32. Leivo $T$, et al. Simultaneous bilateral cataract surgery: economic analysis; Helsinki simultaneous bilateral cataract surgery study report 2. J Cataract Refract Surg. 2011;37(6):1003-8.

33. Lundstrom M, Albrecht S, Roos P. Immediate versus delayed sequential bilateral cataract surgery: an analysis of costs and patient value. Acta Ophthalmol. 2009:87(1):33-8.

34. O'Brien JJ, et al. Immediately sequential bilateral cataract surgery versus delayed sequential bilateral cataract surgery: potential hospital cost savings. Can J Ophthalmol. 2010;45(6):596-601.

35. Rush SW, et al. Prospective analysis of outcomes and economic factors of same-day bilateral cataract surgery in the United States. J Cataract Refract Surg. 2015:41(4):732-9.

36. Malvankar-Mehta MS, et al. Immediately sequential bilateral cataract surgery: a cost-effective procedure. Can J Ophthalmol. 2013:48(6):482-8.

37. Mangione $\mathrm{CM}$, et al. Development of the 25 -item national eye institute visual function questionnaire. Arch Ophthalmol. 2001;119(7):1050-8.

38. Lundstrom M, Pesudovs K. Catquest-9SF patient outcomes questionnaire: nine-item short-form Rasch-scaled revision of the Catquest questionnaire. J Cataract Refract Surg. 2009;35(3):504-13.

39. Versteegh MM, Vermeulen MK, Evers SMAA, de Wit GA, Prenger $R$, Stolk EA. Dutch tariff for the five-level version of EQ-5D. Value Health. 2016;19(4):34352

40. Horsman J, et al. The health utilities index (HUI): concepts, measurement properties and applications. Health Qual Life Outcomes. 2003;1:54.

41. Castor EDC. Castor electronic data capture; 2019. August 28, 2019; Available from: https://castoredc.com.

42. Walker $\mathrm{E}$, Nowacki AS. Understanding equivalence and noninferiority testing. J Gen Intern Med. 2011;26(2):192-6.

43. Piaggio $\mathrm{G}$, et al. Reporting of noninferiority and equivalence randomized trials: extension of the CONSORT 2010 statement. Jama. 2012;308(24):2594604.

44. Hakkaart-van Roijen L, Van der Linden N, Bouwmans C, Kanters T, Tan SS. Kostenhandleiding: methodologie van kostenonderzoek en referentieprijzen voor economische evaluaties in de gezondheidszorg. Diemen: Zorginstituut Nederland; 2015.

45. Sullivan SD, et al. Budget impact analysis-principles of good practice: report of the ISPOR 2012 budget impact analysis good practice II task force. Value Health. 2014;17(1):5-14.

46. Zorginstituut Nederland. Richtlijn voor het uitvoeren van economische evaluaties in de gezondheidszorg. Diemen: Zorginstituut Nederland; 2015.

47. Grzybowski A, Wasinska-Borowiec W, Claoue C. Pros and cons of immediately sequential bilateral cataract surgery (ISBCS). Saudi J Ophthalmol. 2016;30(4):244-9.

48. Henderson BA, Schneider J. Same-day cataract surgery should not be the standard of care for patients with bilateral visually significant cataract. Surv Ophthalmol. 2012;57(6):580-3.

\section{Publisher's Note}

Springer Nature remains neutral with regard to jurisdictional claims in published maps and institutional affiliations. 